\title{
SDU\%
}

\section{Strategic Arrival Times to Queueing Systems}

by

\author{
Jesper Breinbjerg
}

Discussion Papers on Business and Economics

No. $6 / 2016$

FURTHER INFORMATION

Department of Business and Economics Faculty of Business and Social Sciences University of Southern Denmark

Campusvej 55, DK-5230 Odense M

Denmark 


\title{
Strategic Arrival Times to Queueing Systems*
}

\author{
Jesper Breinbjerg ${ }^{\dagger}$
}

September 15, 2016

\begin{abstract}
We examine a non-cooperative queueing game where a finite number of customers seek service at a bottleneck facility which opens at a given point in time. The facility servers one customer at a time on a first-come, first-serve basis and the amount of time required to service each customer is identically and independently distributed according to some general probability distribution. The customers must individually choose when to arrive at the facility, and they prefer to complete service as early as possible, while minimizing the time spent waiting in the queue. These preferences are captured by a general utility function which is decreasing in the waiting time and service completion time of each customer. Applications of such queueing games range from people choosing when to arrive at a grand opening sale to travellers choosing when to line up at the gate when boarding an airplane. We develop a constructive procedure that characterizes an arrival strategy which constitutes a symmetric Nash equilibrium and show that there is at most one symmetric equilibrium. We accompany the equilibrium characterization with numerically computed examples of symmetric equilibria induced by a non-multilinear utility function.
\end{abstract}

Keywords Non-cooperative queueing games $\cdot$ Strategic arrivals $\cdot$ Nash equilibrium

\section{JEL Classification $\quad \mathrm{C} 72 \cdot \mathrm{D} 62 \cdot \mathrm{R} 41$}

${ }^{*}$ The author thanks Lars Peter Østerdal for advise throughout this work and also thanks Refael Hassin, Moshe Haviv, Liron Ravner and Nahum Shimkin for valuable comments. The author also appreciate fruitful discussions with conference and seminar participants at Tel Aviv, Tokyo, Paris and Odense. The financial support from the Danish Council for Independent Research | Social Sciences (Grant ID: DFF-1327-00097) is gratefully acknowledged. Lastly, a special thanks to Refael Hassin and the School of Mathematical Sciences at Tel Aviv University for the warm hospitality provided during my research visit.

${ }^{\dagger}$ Department of Business and Economics, University of Southern Denmark, Campusvej 55, 5230 Odense M, Denmark. Contact: jnb@sam.sdu.dk 


\section{Introduction}

This paper considers the following scenario: A number of customers want to acquire a good or service supplied by a bottleneck facility. The facility is initially closed for service but opens at a commonly known point in time. For the customers to acquire the good or service, they must simultaneously and independently choose a point in time where they submit a request for acquisition. We shall refer to such a submission as an arrival. If the number of arrivals at some point in time exceeds the capacity of the facility, then a queue is created and customers will have to wait in line. The time at which each customer eventually acquires the good or service therefore not only depends on her own arrival time, but also the arrival times of others. As customers might have preferences over outcomes of queueing, the arrival time decision constitutes a non-cooperative queueing game.

There exist many real-life applications of such queueing games. These range from physical queueing situations, e.g. customers choosing when to arrive at a grand opening sale, or travellers choosing when to line up at the gate when boarding an airplane, to virtual queueing situations, e.g. customers choosing when to phone a call centre during opening hours, or foodies choosing when to join an online queue for table reservations at a popular gourmet restaurant.

The strategic choice of arrival times have been extensively studied since the initial formulation of the bottleneck model by Vickrey (1969). A sizable part of this growing body of literature is summarized in a recent survey (Hassin, 2016). The central approach to examine the strategic behavior is to characterize the equilibrium arrival times, presuming that self-optimizing customers make strategic decisions about their arrival. Given the wide range of queueing game applications, various queueing games have been studied which differ in characteristics such as the number of customers, customer preferences over outcomes, the service time required to process a customer, (dis)allowance of arrivals prior to the opening time, etc. In regards to customer preferences, a natural issue to consider is the desire to avoid congestion. This disutility is typically modelled as a waiting time penalty and was first considered by Glazer and Hassin (1983). They investigated the equilibrium arrival times of a (random) number of homogeneous customers who wish to minimize their individual waiting time when the service time requirement of each customer is exponentially distributed. They showed that, in the symmetric equilibrium, each customer arrives according to a continuous probability distribution that extends over a bounded interval of time. This model was further extended to games where the facility has pre-scheduled service times (Glazer and Hassin, 1987), and where customers cannot arrive prior to opening (Hassin and Kleiner, 2011). Moreover, theoretical predictions of related models have been compared to empirical findings in laboratory experiments, which provide support for the symmetric equilibrium solution (Rapoport et al., 2004; Seale et al., 2005; 
Stein et al., 2007). However, customers may not only want to avoid waiting time but also be interested in completing their service at an early time. An example of this could be a commuter driving home from work. He wish to avoid traffic but is not willing to stay at work until midnight in order to achieve this. This additional disutility is often modelled as a lateness penalty that increases the later one completes service. Such preferences was first studied by Jain et al. (2011) who analyzed equilibrium arrival times of a continuum of homogeneous customers. This preference structure was further extended to queueing games with other characteristics, such as systems employing the last-come first-serve rather than the canonical first-come first-serve queue discipline (Platz and Østerdal, 2012), general customer populations with exponentially distributed service times (Juneja and Shimkin, 2013), finite closing times of the facility (Haviv, 2013), and discrete arrival time choices (Breinbjerg et al., 2016).

While the above mentioned studies uncover many important insight into the strategic choices of arrival time in queueing games, they limit their attention to some rather restrictive assumptions regarding customer preferences and service time requirements. Specifically, the preferences are assumed to be represented by a multilinear function of its penalty attribute(s), and moreover, the service time requirement of each customer is often assumed to be either deterministic or exponentially distributed. Both assumptions greatly simplifies the equilibrium analysis and allow for an explicit characterization of the equilibrium arrival times, however, it raises some important questions about the extendability of the equilibrium solution. From a management perspective, it is important to understand and predict the arrival behavior within a large class of queueing games in order to anticipate the arrival incentives induced by a queueing situation.

This paper extends the equilibrium analysis to queueing games with more general classes of customer preferences and service times. Specifically, we focus on queueing games where a finite number of customers, with identical preferences composed of waiting and lateness penalties, arrive to a single-server facility that opens at a given point in time. We shall also restrict attention only to facilities that serve customers on a first-come, first serve (FCFS) basis with no closing time and does not allow customer arrivals prior opening. For this class of queueing games, we provide a constructive procedure for finding a symmetric equilibrium for any game and show that this equilibrium in fact is the only symmetric equilibrium that exist. We note that the basic concept of the constructive procedure carries over to other variants of queueing games that modify the assumptions of closing time, early arrivals, and the priority order discipline ${ }^{1}$. We also provide numerically computed examples of the symmetric equilibrium induced by a non-multilinear utility

\footnotetext{
${ }^{1}$ Breinbjerg and Østerdal (2016) derive equilibrium arrival times under the last-come, first-serve preemptive (LCFS-PR) discipline and numerically compare these with those established in the present paper under the FCFS discipline.
} 
function.

The paper is organized as follows: Section 2 introduces the queueing game and model assumptions. Section 3 first defines the relevant notion of the equilibrium solution (3.1), and thereafter establishes some preliminary results (3.2) used to formulate a constructive procedure for findings such an equilibrium (3.3). Subsequently, some properties of the equilibrium solution are presented and proved (3.4 and 3.5). Section 4 presents some numerical computations of the equilibrium which have been computed via a discretized version of the constructive procedure. We conclude the paper in Section 5 with a brief summary and future research directions. To facilitate an intuitive presentation of the paper, proofs that require technical notation for the transient queueing dynamics are relegated to the Appendix.

\section{The Queueing Game ${ }^{2}$}

A finite set of customers $\mathcal{N}=\{1,2, \ldots, \eta\}, \eta \geq 2$ must be serviced by a single-server facility. The facility admits customers for service within the time interval $\mathbb{R}_{0}^{+}=[0, \infty)$ such that the facility opens for admission at time 0 and does not close before all customers have been served. We assume that the facility serves one customer at a time according to a FCFS regime. Moreover, we assume that the time required for each customer to complete her service is independent and identically distributed according to the cumulative distribution function $S$ which is absolutely continuous, strictly monotonically increasing and has finite moments. If several customers arrive at exactly the same time, then they are admitted in a uniformly randomized order.

Strategy of Arrival. Each customer $i \in \mathcal{N}$ independently choose her time of arrival according to the same (mixed) strategy $F$ which represents a cumulative probability distribution (cdf) that assigns to each point in time $t \in \mathbb{R}_{0}^{+}$the probability $F(t)$ that a customer has arrived by time $t$. We assume that $F: \mathbb{R}_{0}^{+} \rightarrow[0,1]$ where $F$ is nondecreasing, everywhere right-continuous with left limits, and $\lim _{t \rightarrow \infty} F(t)=1$. Let $\mathcal{S}(F)$ denote the support of strategy $F$ which is the smallest closed set of probability 1 , namely $\int_{\mathcal{S}(F)} \mathrm{d} F(t)=1$.

Time of Departure. Given a strategy $F$, we consider the probabilities associated with the time for which a customer has completed her service and departs the system for good. Let $D_{i}$ denote the ex-ante cumulative departure time distribution for any customer $i$ such that $D_{i}(d \mid t, F)$ is the probability that $i$ has departed the system by time $d \in \mathbb{R}_{0}^{+}$given that she arrive at time $t \in \mathbb{R}_{0}^{+}$and the $\eta-1$ other customers arrive according to $F$. Note

\footnotetext{
${ }^{2}$ To facilitate an intuitive formulation of game, we let script letters denote sets or collections, capital letters denote functions, small letters denote set-elements or generic variables, greek letters denote exogenous parameters, and capital bold letters denote random variables.
} 
that $\lim _{d \rightarrow \infty} D_{i}(d \mid t, F)=1$ for all $t$ since FCFS is work-conserving ${ }^{3}$. Note also that $D_{i}(d \mid t, F)=0$ for all $d \leq t$. A formal definition of $D_{i}$ is given in Appendix A.1 using standard queueing relations.

Utility Function. We assume that the customers have identical preferences. Each customer wants to receive service as early as possible and spend a minimum of time in the queue. To capture such preferences, let $U$ denote a utility function such that the value $U(t, d)$ is the utility of a customer who arrives at time $t$ and departs the system at time $d$ after waiting in the queue for $d-t$ time units. We assume that $U$ is well-defined and continuous at all $d \geq t$, and strictly decreasing in both the departure time $d$ and the waiting time $d-t$. Moreover, $U$ is bounded from above and $\lim _{t \rightarrow \infty} U(t, t)=-\infty$.

We assume that every customer is (von Neumann-Morgenstern) rational and aims to maximize her expected utility with respect to her time of arrival. For a given strategy $F$, we denote by $U_{i}$ customer $i$ 's expected utility by arriving at time $t$ with certainty when the $\eta-1$ other customers arrive according to $F$, so

$$
U_{i}(t, F)=\int_{t}^{\infty} U(t, d) \mathrm{d} D_{i}(d \mid t, F)
$$

where $\int$ is the Lebesgue integral over the cumulative departure time distribution $D_{i}$.

A queueing game is a tuple $\mathcal{G}=\langle\eta, U, S\rangle$ where $\eta$ is the finite number of customers, $U$ is the utility function of each customer, and $S$ is the independent and identical customer service time distribution.

\section{Equilibrium Analysis}

This section first defines the notion of an symmetric equilibrium in Section 3.1, which is used as the solution concept to study outcomes of the queueing game $\mathcal{G}$. We next provide preliminary insights in Section 3.2 that will be useful for the foregoing analysis. Section 3.3 presents a constructive procedure for finding an equilibrium, and lastly, Section 3.4 and 3.5 presents and proves some general equilibrium properties, respectively.

\subsection{Symmetric Equilibrium}

To study the strategic arrivals of customers in a queueing game $\mathcal{G}$, we adopt the Nash equilibrium concept for which no customer can improve her expected utility by a unilateral change of her time of arrival (Nash, 1951). Formally, we define the equilibrium as follows,

\footnotetext{
${ }^{3}$ A queueing discipline is work-conserving if the server is never idle when the queue is not empty (see Hassin and Haviv, 2003).
} 
Definition 1 For any queueing game $\mathcal{G}$, we say that strategy $F$ constitutes a symmetric (Nash) equilibrium if, and only if, it holds for every customer $i \in \mathcal{N}$ that

(i) $U_{i}(t, F) \geq U_{i}(s, F)$ for all $t \in \mathcal{S}(F)$ and $s \in \mathbb{R}_{0}^{+}$

(ii) $U_{i}(t, F)=U_{i}(s, F)$ for all $t, s \in \mathcal{S}(F)$.

Definition 1 prescribes a probability distribution that assigns to every point in time, the probability of each customer arriving at the facility, such that no customer wishes to change her strategy unilaterally. We shall occasionally refer to a strategy that constitutes a symmetric equilibrium as a equilibrium strategy.

The interpretation of an equilibrium strategy is often considered more as a descriptive rather than prescriptive solution for real-life customer behavior. In this context, we therefore do not necessarily expect that each customer fully randomize her arrival decision according to a probability distribution in real-life queueing situations, but that this distribution rather reflects her beliefs about the others customers decisions. These beliefs may be the result of repeated interaction and learning within everyday queueing situations.

\subsection{Preliminaries}

The first result states some important properties of the cumulative departure distribution.

Proposition 1 For any queueing game $\mathcal{G}$ and any customer $i \in \mathcal{N}$, the cumulative departure time distribution $D_{i}$ satisfies the following properties for any given $t \in \mathbb{R}_{0}^{+}$and strategy F:

(i) $D_{i}(d \mid t, F)$ is continuous over $\mathbb{R}_{0}^{+}$.

(ii) If $F(t)>\lim _{s \uparrow t} F(s)$, then $D_{i}(d \mid t, F) \leq \lim _{s \uparrow t} D_{i}(d \mid s, F)$ for all $d \in \mathbb{R}_{0}^{+}$with strict inequality at some $d$.

(iii) If $F(0)>0$, then $D_{i}\left(d \mid 0, \mathcal{F}_{-i}\right) \geq \lim _{s \downarrow 0} D_{i}\left(d \mid s, \mathcal{F}_{-i}\right)$ for all $d \in \mathbb{R}_{0}^{+}$with strict inequality at some $d$.

(iv) Let $\tilde{F}$ be another strategy such that $\tilde{F}(s)=F(s)$ for all $0 \leq s<t$ and $\tilde{F}(t)=$ $\lim _{s \uparrow t} \tilde{F}(s)+\tilde{x}$. If $F(t)=\lim _{s \uparrow t} F(s)+x$, then for all $0 \leq x<\tilde{x}$, it holds that $D_{i}(d \mid t, F) \geq D_{i}(d \mid t, \tilde{F})$ for all $d \in \mathbb{R}_{0}^{+}$with strict inequality at some $d$.

The proof of Proposition 1 is relegated to Appendix A.1 as it requires additional notation to describe the stochastic queueing process and its sample path relations. The claims of Proposition 1 are quite intuitive. That is, a customer's departure time distribution is continuous since every customer's service time distribution $S$ is continuous [claim (i)]. A customer that arrives at a point in time where other customers may arrive simultaneously (i.e. a point of discontinuity in $F$ ), has a lower probability of having departed the system at any point time than if she instead arrive immediately before such point and avoid 
the excess congestion [claim (ii)]. If other customers may arrive at opening time, then a customer who arrives immediately after opening time has a lower probability of having departed the system at any point in time than if she instead arrived at the opening time [claim (iii)]. Lastly, if a customer arrives at a point of discontinuity, then the probability of a customer having departed the system at any point in time is decreasing in the size of the upward jump [claim (iv)].

The next result addresses for any customer $i$ the relationship between $U_{i}$ and $D_{i}$ and is a corollary of Rothschild and Stiglitz (1970)'s Equivalence Theorem.

Lemma 1 For any queueing game $\mathcal{G}$ and any customer $i \in \mathcal{N}$, let $F$ and $\tilde{F}$ be two distinct strategies. Then $U_{i}(t, F) \geq U_{i}(t, \tilde{F})$ for any $t \in \mathbb{R}_{0}^{+}$if $D_{i}(d \mid t, F) \geq D_{i}(d \mid t, \tilde{F})$ for all $d \in \mathbb{R}_{0}^{+}$. Furthermore, if strict inequality holds for some $d$, then $U_{i}(t, F)>U_{i}(t, \tilde{F})$.

Note that Lemma 1 is a corollary of a "dual" statement of the Equivalence Theorem in the sense that the present paper considers strictly monotonically decreasing utility functions whereas Rothschild and Stiglitz stated their result wrt. non-decreasing utility functions.

\subsection{A Constructive Procedure}

This section presents a method for constructing a strategy that constitutes a symmetric equilibrium of a queuing game $\mathcal{G}$. Moreover, the procedure also suffices as a constructive proof for the existence of a symmetric equilibrium strategy. The main result is summarized as follows,

Theorem 1 One has a constructive procedure for finding a symmetric equilibrium of any queueing game $\mathcal{G}$.

We prove this claim by defining a family of functions $\left\{X_{l}\right\}_{0 \leq l \leq 1}$ where for each $l, X_{l}$ is the limit of a convergent and recursive sequence $\left\{\bar{X}_{l, h} \mid 0 \leq l \leq 1\right\}_{h \in \mathbb{N}}$ indexed by the nonnegative integer $h$. We then show that a member of the family $\left\{X_{l}\right\}$ in fact represents a strategy that constitutes a symmetric equilibrium.

We start by providing some useful notation. For any given $l \in[0,1]$ and $h \in \mathbb{N}$, let $X_{l, h}: \mathbb{R}_{+}^{0} \rightarrow[0,1]$ be a function where $X_{l, h}(t)$ is the image of $X_{l, h}$ at $t$. For any point in time $t$ where $X_{l, h}(t) \leq 1$ and $X_{l, h}$ is non-decreasing and right-continuous over $[0, t]$, then the expected utility $U_{i}\left(t, X_{l, h}\right)$ is well-defined for any customer $i \in \mathcal{N}$.

Fix $l$ to be a constant, $0 \leq l \leq 1$, and assume that $X_{l, h}(0)=l$ for all $h$. Intuitively, one may think of $X_{l, h}(0)$ as the expected share of $\eta-1$ customers that arrive at time 0 . Note that customer $i$ 's expected utility of arriving at time 0 when the other $\eta-1$ customers arrive according to $X_{l, h}$ is given by $U_{i}\left(0, X_{l, h}\right)$.

We now define a sequence of recursive functions $X_{l, 0}, X_{l, 1}, X_{l, 2}, \ldots$ where $X_{l, 0}$ is the designated starting term. We start by characterizing for $h=0$ the properties of $X_{l, 0}$. For 
each $t \in \mathbb{R}_{0}^{+}$, let

$$
X_{l, 0}(t)= \begin{cases}l & \text { for } x_{l, 0}^{t}<0 \\ l+x_{l, 0}^{t} & \text { for } x_{l, 0}^{t} \in[0,1-l] \\ 1 & \text { for } x_{l, 0}^{t}>1-l\end{cases}
$$

where

$$
x_{l, 0}^{t}=\sup \left\{\begin{array}{l|l}
x \in \mathbb{R}_{0}^{+} & \begin{array}{l}
U_{i}\left(0, X_{l, 0}\right) \leq \lim _{s \downarrow t} U_{i}\left(s, X_{l, 0}^{x}\right) \\
\text { where } X_{l, 0}^{x}(t)=l+x \text { and } \\
X_{l, 0}^{x}(s)=l \text { for all } 0 \leq s<t
\end{array}
\end{array}\right\} .
$$

Intuitively speaking, $x_{l, 0}^{t}$ represents the maximal expected share of $\eta-1$ customers that arrive exactly at time $t$ such that a customer who arrives immediately after $t$ yields at least the same expected utility as she would if she instead arrives at time 0 , given that no customers arrive in the interval $(0, t)$. By Proposition 1, part $(i v)$, it follows that $x_{l, 0}^{t}$ is uniquely determined for each $t$ since $\lim _{s \downarrow t} U_{i}\left(t, X_{l, 0}^{x}\right)$ is strictly decreasing in $x$. Note that $X_{l, 0}$ is right-continuous at any $t$ since $U_{i}$ is continuous by Proposition 1, part $(i)$. Note also that $X_{l, 0}$ is bounded and may not necessarily be monotonically increasing. For each $t$, let

$$
\bar{X}_{l, 0}(t)=\max \left\{X_{l, 0}(s) \mid 0 \leq s \leq t\right\}
$$

By construction, $\bar{X}_{l, 0}$ is monotonically increasing over $\mathbb{R}_{0}^{+}$. Figure 1 graphically illustrates an example of $X_{l, 0}$ and $\bar{X}_{l, 0}$.

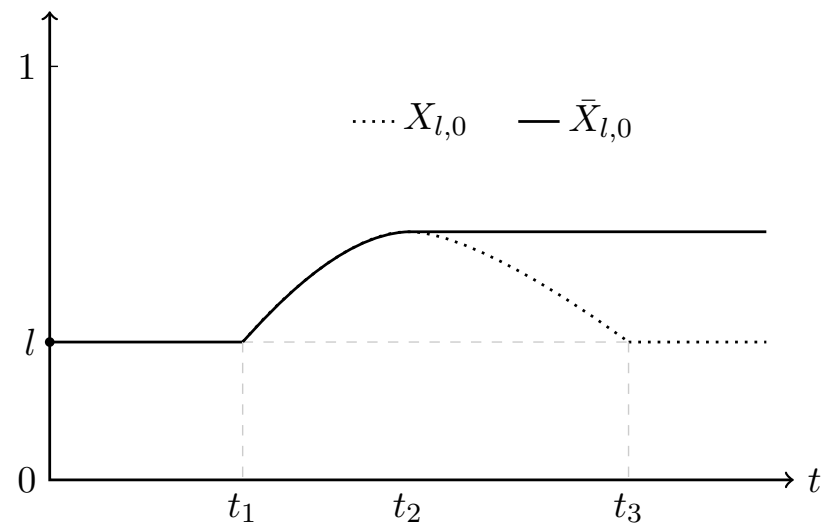

Figure 1: Example of $X_{l, 0}$ and $\bar{X}_{l, 0}: l$ is the expected share of customers that arrives at time 0 . The function $X_{l, 0}$ is increasing over the time interval $\left[t_{1}, t_{2}\right]$, hence $\bar{X}_{l, 0}$ is also increasing. Conversely, $X_{l, 0}$ is decreasing over $\left(t_{2}, t_{3}\right]$, while $\bar{X}_{l, 0}$ remains the maximum value $X_{l, 0}$ obtained at time $t_{2}$. Lastly, $X_{l, 0}(t)=l$ for all $t>t_{3}$. In this example, $X_{l, 0}$ is a concave function, however, this may not necessarily be the case for all utility functions $U$.

We next characterize the recursive statement of $X_{l, h}$ for each $h>0$. Suppose that 
$\bar{X}_{l, h-1}$ has been defined for $h>0$. For each $t \in \mathbb{R}_{0}^{+}$, let

$$
X_{l, h}(t)= \begin{cases}\bar{X}_{l, h-1}(t) & \text { for } x_{l, h}^{t}<0 \\ \bar{X}_{l, h-1}(t)+x_{l, h}^{t} & \text { for } x_{l, h}^{t} \in\left[0,1-\bar{X}_{l, h-1}(t)\right] \\ 1 & \text { for } x_{l, h}^{t}>1-\bar{X}_{l, h-1}(t)\end{cases}
$$

where

$$
x_{l, h}^{t}=\sup \left\{\begin{array}{l|l}
x \in \mathbb{R}_{0}^{+} & \begin{array}{l}
U_{i}\left(0, X_{l, h}\right) \leq \lim _{s \downarrow t} U_{i}\left(s, X_{l, h}^{x}\right) \text { where } \\
X_{l, h}^{x}(t)=\bar{X}_{l, h-1}(t)+x \text { and } \\
X_{l, h}^{x}(s)=\bar{X}_{l, h-1}(t) \text { for all } 0 \leq s<t
\end{array}
\end{array} .\right.
$$

Intuitively speaking, $x_{l, h}^{t}$ represents the maximal expected share of $\eta-1$ customers that arrive exactly at time $t$ such that a customer who arrives immediately after $t$ yields at least the same expected utility as she would if she instead arrives at time 0 , given that the $\eta-1$ customers arrive according to $\bar{X}_{l, h-1}$. By Proposition 1 , part $(i v)$, it follows that $x_{l, h}^{t}$ is uniquely determined for each $t$ since $\lim _{s \downarrow t} U_{i}\left(t, \mathcal{X}_{l, h}^{x}\right)$ is strictly decreasing in $x$. Note that $X_{l, h}$ is right-continuous at any $t$ since $U_{i}$ is continuous by Proposition 1 , part ( $i$ ) Note also that $X_{l, h}$ is bounded and may not necessarily be monotonically increasing. For each $t$, let

$$
\bar{X}_{l, h}(t)=\max \left\{X_{l, h}(s) \mid 0 \leq s \leq t\right\}
$$

By construction, $\bar{X}_{l, h}$ is monotonically increasing.

The recursive process yields the sequence $\bar{X}_{l, 0}, \bar{X}_{l, 1}, \ldots$ which is bounded and monotonically increasing with $\bar{X}_{l, 0}(t) \leq \bar{X}_{l, 1}(t) \leq \ldots$ over $h \in \mathbb{N}$ and for all $t \in \mathbb{R}_{0}^{+}$. It thus follows by the monotone convergence theorem that the sequence is convergent. Let $X_{l}(t)=\lim _{h \rightarrow \infty} \bar{X}_{l, h}(t)$ denote the limit of the sequence for each $t$. Figure 2 graphically illustrates an example of a recursive sequence $\bar{X}_{l, 0}, \bar{X}_{l, 1}, \ldots$ that converges towards a limit $X_{l}$.

So far $l$ has been fixed. We now define a family of functions $\left\{X_{l}\right\}_{0 \leq l \leq 1}$ where for each $l, X_{l}$ is the limit of the convergent and recursive sequence $\left\{\bar{X}_{l, h} \mid 0<l \leq 1\right\}_{h \in \mathbb{N}}$. For each member of $\left\{X_{l}\right\}$, we examine whether it represents an equilibrium strategy. Specifically, we establish a value of $l$ for which the member of $\left\{X_{l}\right\}$ satisfies the following three criteria: (1) $X_{l}$ is non-decreasing and right-continuous with left limits over $\mathbb{R}_{0}^{+},(2) \lim _{t \rightarrow \infty} X_{l}(t)=1$, and (3) for any customer $i, U_{i}\left(t, X_{l}\right) \geq U_{i}\left(s, X_{l}\right)$ for each $t$ where $X_{l}(t)>X_{l}(s)$ for all $0 \leq s<t$, and moreover, $U_{i}\left(t_{b}^{l}, X_{l}\right) \geq U_{i}\left(q, X_{l}\right)$ for all $q \geq t_{b}^{l}$ where $t_{b}^{l}=\inf \left\{t \mid X_{l}(t)=1\right\} .{ }^{4}$

\footnotetext{
${ }^{4}$ Note that property (3) is alternative statement of Definition 1 where $X_{l}$ need not have a well-defined support set $\mathcal{S}$. Intuitively, property (3)'s first term states that a customer obtains at least the same expected utility by arriving at any point in time where $X_{I}$ is not "flat" compared to that of arriving at any point in time. The second term states that no customer can obtain a strictly higher expected utility by arriving later than the point in time $t_{b}^{l}$.
} 


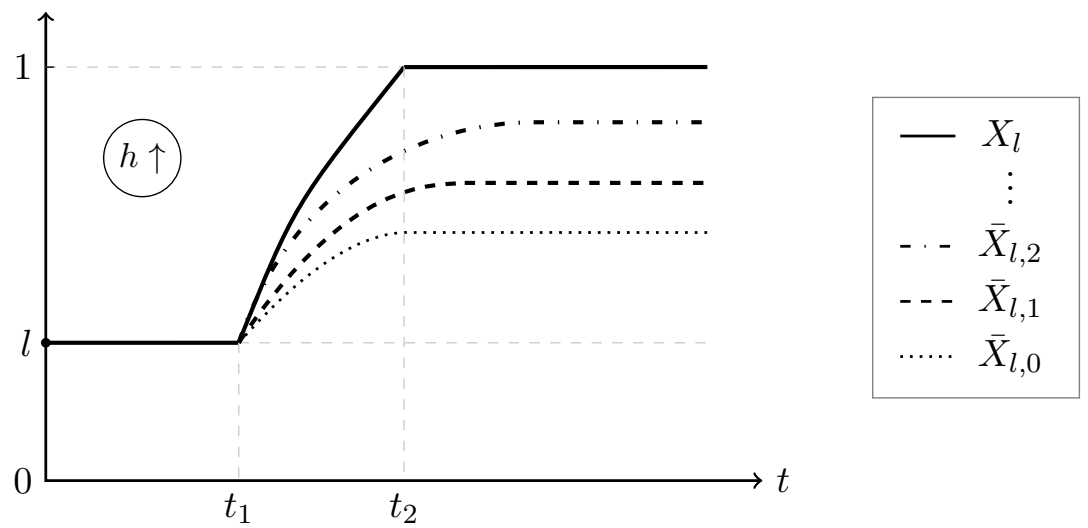

Figure 2: Example of recursive sequence $\bar{X}_{l, 0}, \bar{X}_{l, 1}, \ldots$ : As the number of iterations $h$ increases, the $h$ th recursively stated term $\bar{X}_{l, h}$ converge towards the limit $X_{l}$. In this example, there exists a point in time $t_{2}$ for which $X_{l}\left(t_{2}\right)=1$. However, this is not necessarily the case for all values of $l$.

We first note that $X_{l}$ satisfy criteria (1) for all $0 \leq l \leq 1$, by construction. We then note that $X_{l}$ cannot represent an equilibrium strategy for values of $l$ where $t_{b}^{l}=\infty$ as criteria (2) is thus not satisfied. Cases for which $t_{b}^{l}=\infty$ might occur for sufficiently low values of $l$. In what follows, we therefore examine members of $\left\{X_{l}\right\}$ for decreasing values of $l$ starting from 1 .

We start by examining the member of $\left\{X_{l}\right\}$ for which $l=1$. We consider two distinct cases: Either $U_{i}\left(t, X_{1}\right) \leq U_{i}\left(0, X_{1}\right)$ for all $t>0$ and any $i$ and we conclude that $X_{1}$ satisfy criteria (1) through (3) and thus represents an equilibrium strategy. Otherwise, $U_{i}\left(t, X_{1}\right)>U_{i}\left(0, X_{1}\right)$ for at least one point $t>0$ and $X_{1}$ cannot represent an equilibrium strategy. In the latter case, we proceed to members of $\left\{X_{l}\right\}$ with lower values of $l$. We make the following observations:

(a) There exists some $\epsilon>0$ (sufficiently small) such that the member of $\left\{X_{l}\right\}$ for $l=1-\epsilon$ have $t_{b}^{l}<\infty$. This follows immediately by construction of $X_{l}$, once we note that $U_{i}\left(t, X_{1}\right)>U_{i}\left(0, X_{1}\right)$ for some $t>0$.

(b) For all members of $\left\{X_{l}\right\}$ where $t_{b}^{l}<\infty$ then $t_{b}^{l}$ is continuous at $l$. This follows by construction of $X_{l}$.

Combining (a) and (b), it follows that there must exist an $l=l^{*}$ such that $X_{l^{*}}$ satisfy criteria (3). It thus immediately follows that $X_{l^{*}}$ represents a strategy that constitutes a symmetric equilibrium. Figure 3 graphically illustrates an example of such $X_{l^{*}}$.

\subsection{Equilibrium Properties}

This section establishes some general properties of an equilibrium strategy. The properties are summarized in the following theorem: 


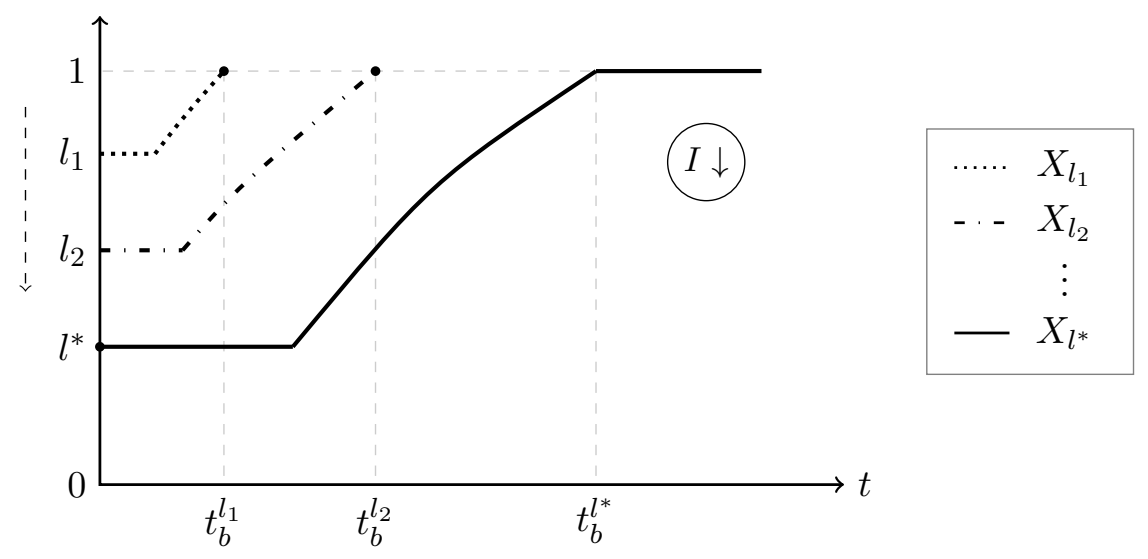

Figure 3: Example of $\left\{X_{l}\right\}_{0 \leq l \leq 1}$ : All members of $\left\{X_{l}\right\}$ for which $l^{*} \leq l \leq 1$ yields $U_{i}\left(t_{b}^{l}, X_{l}\right)<U_{i}\left(s, X_{l}\right)$ for $s>t_{b}^{l}$ whereas the member of $\left\{X_{l}\right\}_{0<l \leq 1}$ with $l=l^{*}$ represents an equilibrium strategy.

Theorem 2 For any queueing game $\mathcal{G}$, there exists one, and only one, strategy that constitutes a symmetric equilibrium. Let $F$ be a symmetric equilibrium strategy, then the following properties holds for F:

(i) $F(t)$ is continuous at all $t>0$ and has $0<F(0) \leq 1$.

(ii) The support $\mathcal{S}(F)$ of $F$ is a compact set and there exists a non-empty and bounded interval $\left(0, t_{a}\right)$ such that $\mathcal{S}(F) \cap\left(0, t_{a}\right)=\emptyset$ for $0<t_{a} \leq \infty$.

Figure 4 illustrates an example of the properties of an equilibrium strategy. In this example, customers assign a strictly positive probability $F(0)>0$ for arriving at the opening time 0 . Immediately after opening, no customer arrives at the facility within the interval of time $\left(0, t_{a}\right)$, i.e. $F(t)=F(0)$ for all $0<t<t_{a}$. At time $t_{a}$ and onwards, the customers then smoothly assigns probability of arrival up until some time $t_{b}<\infty$ at which point all customers have arrived.

Theorem 2 suggests that there may be queueing games for which the equilibrium strategy has slightly different characteristics than that illustrated in Figure 4. That is, some games may induce an equilibrium strategy $F$ for which $F(0)=1$ such that $\mathcal{S}(F)=$ $\{0\}$. Conversely, other games may induce an equilibrium strategy $F$ for which $0<F(0) \leq$ 1 and $\mathcal{S}(F) \cap\left(0, t_{a}\right)=\emptyset$ for $t_{a}<\infty$ (similar to that of Figure 4 ) but where $F$ is not necessarily strictly monotonic in $\left[t_{a}, t_{b}\right]$ for $t_{b}=\inf \{t \mid F(t)=1\}<\infty$, i.e. $\mathcal{S}(F) \backslash\{0\}$ is not necessarily a connected set. Specifically, $\mathcal{S}(F) \backslash\{0\}$ may be a finite union of disjoint closed intervals, which graphically means that the arrival distribution has intervals within $\left[t_{a}, t_{b}\right]$ where it is "flat".

\subsection{Proof of Theorem 2}

This section is devoted to the proof of Theorem 2 which proceeds through several lemmas. Specifically, we start by establishing some important characteristics of an equilibrium 


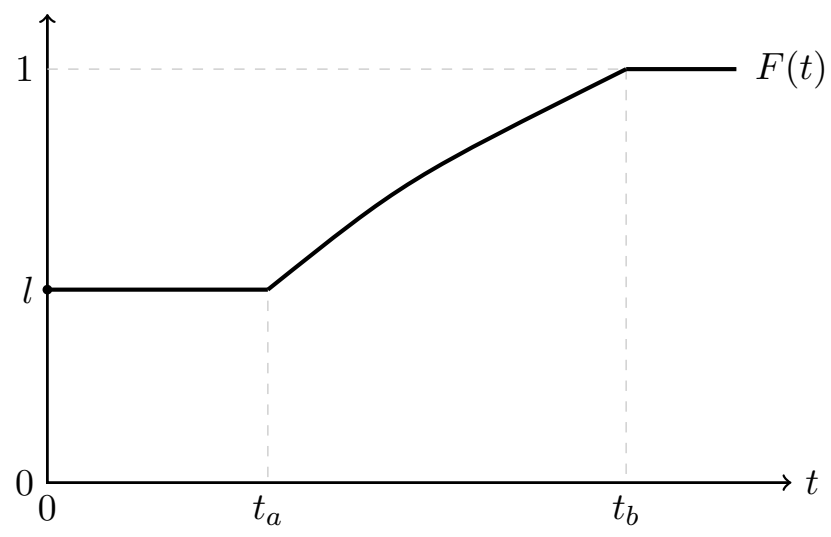

Figure 4: Example of an equilibrium strategy: Let $F$ be a strategy that constitutes a symmetric equilibrium. In this example, $F$ has $F(0)=l$ for $0<l<1$, and has the support $\mathcal{S}(F)=\{0\} \cup\left[t_{a}, t_{b}\right]$ for $t_{b}<\infty$ such that $F$ is strictly monotonic over $\left[t_{a}, t_{b}\right]$. By definition, $U_{i}\left(t, \mathcal{F}_{-i}\right)=U_{i}\left(t^{\prime}, \mathcal{F}_{-i}\right)$ for all $t, t^{\prime} \in \mathcal{S}(F)$ and any $i$.

strategy through Lemma 2 to 5, and subsequently, show that there exist a most one strategy that constitutes a symmetric equilibrium by Lemma 6 and 7 .

The first result establishes that every customer assigns a strictly positive probability of arriving at the opening time 0 .

Lemma 2 Let $F$ be an equilibrium strategy for some queueing game $\mathcal{G}$. Then $F(0)>0$.

Proof. Suppose that $F(0)=0$. Then any customer $i$ could arrive at time 0 and start service immediately without waiting. That is, $U_{i}(0, F)>U_{i}(t, F)$ for all $t>0$. This contradicts the equilibrium definition and thus proves that $F(0)>0$ in equilibrium.

The next result addresses the continuity of an equilibrium strategy.

Lemma 3 Let $F$ be an equilibrium strategy for some queueing game $\mathcal{G}$. Then there exists no time $t>0$ such that $F(t)>\lim _{s \uparrow t} F(s)$.

Proof. We prove by contradiction. Suppose that $F(t)>\lim _{s \uparrow t} F(s)$ for some point in time $t \mathbb{R}_{0}^{+}$. Then it follows from Proposition 1, part (ii) that $D_{i}(d \mid t, F) \leq \lim _{s \uparrow t} D_{i}(d \mid s, F)$ for all $d \in \mathbb{R}_{0}^{+}$with strict inequality at some $d$; hence $U(t, F)<\lim _{s \uparrow t} U(s, F)$ by Lemma 1. This contradicts the equilibrium assumption and thus proves that $F(t)=\lim _{s \uparrow t} F(s)$ for all $t$.

Since any strategy $F$ by definition is everywhere right-continuous, then Lemma 2 and 3 immediately proves Part $(i)$ of Theorem 2.

The next result establishes that every customer arrives at the facility with certainty within some bounded interval of time. 
Lemma 4 Let $F$ be an equilibrium strategy for some queueing game $\mathcal{G}$. Then $\mathcal{S}(F)$ is a bounded set.

Proof. It follows immediately from Lemma 2 that $\mathcal{S}(F)$ is bounded from below at 0 . We next prove by contradiction that $\mathcal{S}(F)$ is also bounded from above. Suppose that $\inf \{t \mid F(t)=1\}=\infty$. For any customer $i \in \mathcal{N}$, it then follows by the equilibrium definition that $U_{i}(t, F)=-\infty$ for all $t \in \mathcal{S}(F)$ since $\lim _{t \rightarrow \infty} D_{i}(d \mid t, F)=0$ for all $d<\infty$ and $\lim _{t \rightarrow \infty} U(t, t)=-\infty$, by definition. This leads to a contradiction, however, since customer $i$ obtains $U_{i}(s, F)>-\infty$ by arriving at $s<\infty$ since $\min \left\{d \mid D_{i}(d \mid s, F)=1\right\}<$ $\infty$ under the work-conserving FCFS discipline. This proves that $\mathcal{S}(F)$ must be bounded from above and therefore bounded, hence proving Lemma 4.

Since the support $\mathcal{S}(F)$ is closed, by definition, it immediately follows from the HeineBorel theorem that $\mathcal{S}(F)$ is a compact set.

The following lemma relates to the interior "hole" in the support of an equilibrium strategy.

Lemma 5 Let $F$ be an equilibrium strategy for some queueing game $\mathcal{G}$. Then there exists a non-empty and bounded interval of time $\left(0, t_{a}\right)$ such that $\mathcal{S}(F) \cap\left(0, t_{a}\right)=\emptyset$ for $0<t_{a} \leq \infty$.

Proof. If follows by Lemma 2 that $F(0)>0$. By Proposition 1, part (iii), it then follows for any $i \in \mathcal{N}$ that $D_{i}(d \mid 0, F) \geq \lim _{s \downarrow 0} D_{i}(d \mid s, F)$ for all $d \in \mathbb{R}_{0}^{+}$with strict inequality at some $d$, hence $U_{i}(0, F)>\lim _{s \downarrow 0} U_{i}(s, F)$ by Lemma 1 . As any customer that arrives immediately after time zero needs to wait for the service completion of the customers who arrived at time zero according to the FCFS regime, the inequality of expected utility extends to the interval $\left(0, t_{a}\right)$ where $t_{a}=\inf \left\{t>0 \mid U_{i}(t, F) \geq U_{i}(0, F)\right\}$ in the sense that $D_{i}(d \mid 0, F) \geq D_{i}(d \mid t, F)$ for any $t \in\left(0, t_{a}\right)$ and all $d$ with strict inequality at some $d$, hence $U_{i}(0, F)>U_{i}(t, F)$ for all $t \in\left(0, t_{a}\right)$. Note that $t_{a}=\infty$ when $\left\{t>0 \mid U_{i}(t, F) \geq\right.$ $\left.U_{i}(0, F)\right\}=\emptyset$ since $\inf \emptyset=\infty$. This proves that the interval $\left(0, t_{a}\right)$ for $0<t_{a} \leq \infty$ cannot intersect with the support $\mathcal{S}(F)$ as this otherwise would contradict the equilibrium definition.

Lemma 4 and 5 immediately prove Theorem 2, part (ii).

The next results establishes some properties of the cumulative departure time distribution $D_{i}$ under different strategies. The results are key for later establishing the uniqueness of an equilibrium strategy.

Lemma 6 Let $F$ and $\tilde{F}$ be two distinct equilibrium strategies for some queueing game $\mathcal{G}$. For any customer $i \in \mathcal{N}$, then the following properties holds for the cumulative departure time distribution $D_{i}$ : 
(i) If $F(0)=\tilde{F}(0)$ and $F\left(s_{2}\right)-F\left(s_{1}\right) \leq \tilde{F}\left(s_{2}\right)-\tilde{F}\left(s_{1}\right)$ for all $0 \leq s_{1}<s_{2} \leq t$ with strict inequality for some $s_{1}$ and $s_{2}$. Then $D_{i}(d \mid t, F) \geq D_{i}(d \mid t, \tilde{F})$ for all $d \in \mathbb{R}_{0}^{+}$ with strict inequality at some $d$.

(ii) If $F(t)=\tilde{F}(t)$ and $F(s) \leq \tilde{F}(s)$ for all $0 \leq s \leq t$ with strict inequality at some $s$. Then $D_{i}(d \mid t, F) \leq D_{i}(d \mid t, \tilde{F})$ for all $d \in \mathbb{R}_{0}^{+}$with strict inequality at some $d$.

The claim follows by stochastic coupling arguments which are presented in Appendix A.2.

The next result establish the uniqueness of an equilibrium strategy.

Lemma 7 There exist at most one strategy $F$ that constitutes a symmetric equilibrium for a queueing game $\mathcal{G}$.

Proof. We prove by contradiction. Let $F$ and $\tilde{F}$ be two distinct equilibrium strategies such that $F \neq \tilde{F}$. Let $t_{b}=\min \{t \mid F(t)=1\}$ and $\tilde{t}_{b}=\min \{t \mid \tilde{F}(t)=1\}$. In what follows, we distinguish between the following three cases of $F(0)$ and $\tilde{F}(0)$ :

$F(0)<\tilde{F}(0)$ : In this case, it follows immediately that $U_{i}(t, F)>U_{i}(t, \tilde{F})$ for all $t \in \mathcal{S}(F)$ and any $i \in \mathcal{N}$. We further distinguish between the following cases of $t_{b}$ and $\tilde{t}_{b}$.

(i) $t_{b}>\tilde{t}_{b}$ : In this case, $\tilde{F}(t)$ dominates $F(t)$ in the sense that $\tilde{F}(t) \geq F(t)$ for all $0 \leq t \leq \tilde{t}_{b}$ with strict inequality at some $t$. However, this leads to a contradiction since any customer arriving in accordance to distribution $\tilde{F}$ could arrive with certainty immediately after $t_{b}$ and obtain a higher expected utility.

(ii) $t_{b} \leq \tilde{t}_{b}:$ In this case, there exists a point in time where $F$ and $\tilde{F}$ intersect. Let $s=\min \{t \mid F(t)=\tilde{F}(t)\}$ denote the earliest point for which this is the case. It thus follows immediately from Lemma 6 , part $(i i)$, that $U_{i}(s, F)<U_{i}(s, \tilde{F})$ since $F(s)=\tilde{F}(s)$ and $F_{i}(t) \leq \tilde{F}_{i}(t)$ for all $0 \leq t \leq s$. This leads to a contradiction and thus proves that $F$ and $\tilde{F}$ cannot both be an equilibrium strategy.

$F(0)>\tilde{F}(0)$ : The case is symmetric to that of $F(0)<\tilde{F}(0)$ and thus omitted.

$F(0)=\tilde{F}(0)$ : In this case, it follows that $U_{i}(t, F)=U_{i}(t, \tilde{F})$ for all $t \in \mathcal{S}(F)$. Given that $F \neq \tilde{F}$, then one of the following cases must be true:

(i) $t_{b} \geq \tilde{t}_{b}$ : Let $s=\max \left\{t \mid F(t)=\tilde{F}(t), 0 \leq t<\tilde{t}_{b}\right\}$ be the latest point in time at which the expected share of arrived customers is the same for the two arrival distributions. Note that $s$ is well-defined since $F$ and $\tilde{F}$ are continuous and $\tilde{F}\left(\tilde{t}_{b}\right) \geq F\left(t_{b}\right)$. Then there must exists an $\epsilon>0$ (sufficiently small) such that $F(s+\epsilon)-F(s)<\tilde{F}(s+\epsilon)-\tilde{F}(s)$. In this case, It follows from Lemma 6, part $(i)$, that $U_{i}(s+\epsilon, F)>U_{i}(s+\epsilon, \tilde{F})$. Hence, this contradicts that $F$ and $\tilde{F}$ provides the same expected utility and proves that $F$ and $\tilde{F}$ cannot both be an equilibrium strategy. 
(ii) $t_{b}<\tilde{t}_{b}$ : The case is symmetric to that of $t_{b} \geq \tilde{t}_{b}$ and thus omitted.

With Theorem 1 proving the existence of a symmetric equilibrium strategy, Lemma 7 completes the proof of Theorem 2.

\section{Numerical Computation}

This section demonstrates some numerically computed equilibrium strategies for various queueing games. Specifically, we assume that customer service times are i.i.d. exponentially distributed and compute equilibrium strategies for an example of a non-linear utility function.

The numerical procedure used to compute an equilibrium strategy is a discretized variant of the constructive procedure in Section 3.3. Figure 5 illustrates a flowchart of the numerical procedure. For a given set of inputs, the procedure performs a binary search of the value of $l$ that induce a function $X_{l}$ that represents an equilibrium strategy.

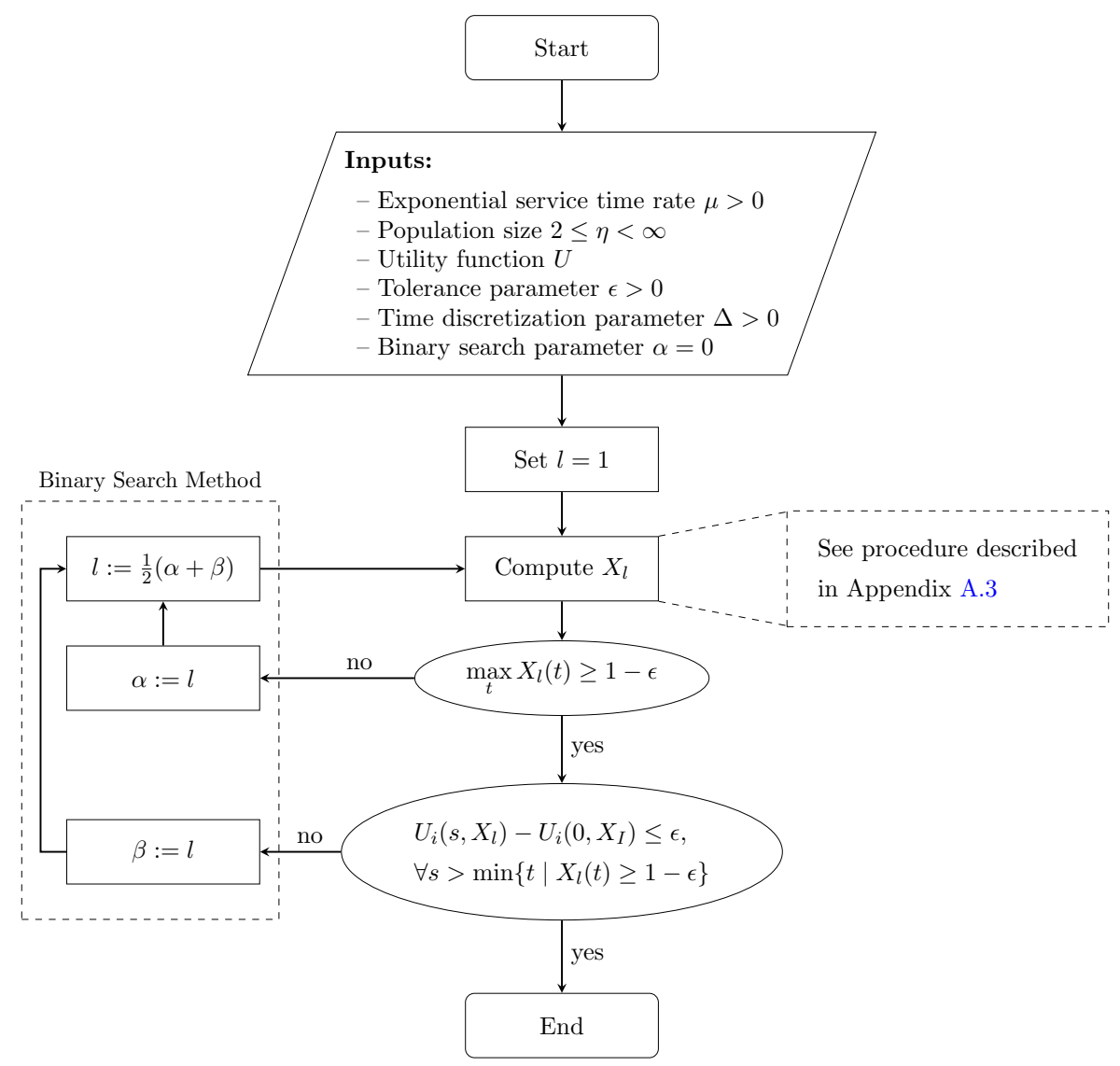

Figure 5: Flowchart of the numerical procedure: Each geometric shape represents an action within the procedure: The rounded squares are the start and ending, the trapezium is the exogenous inputs, the squares are steps in the process, and circles are binary decisions (yes/no) based on a question. The arrows indicate the flow from one process or decision to another. Note that $:=$ is the assignment operator that changes an existing variable's value. 
As indicated in Figure 5, the numerical procedure used to compute $X_{l}$ for a given value of $l$ and its computational complexity is described in detail in Appendix A.3. Note that the number of required iterations for the binary search of $l$ to converge is a function of the tolerance parameter $\epsilon$. For any equilibrium strategy with $l<1$, the procedure requires multiple (possibly many) search iterations of $l$ before convergence.

We applied the numerical procedure presented above to compute equilibrium strategies for several examples of customer population size which is depicted in Figure $6 .^{5}$ For a population size of two customers, the computed equilibrium strategy prescribes that both customers arrive with certainty at the opening time 0. As the population size increases, both the time interval immediately after opening in which no customer arrive and the strategies support set increases monotonically.

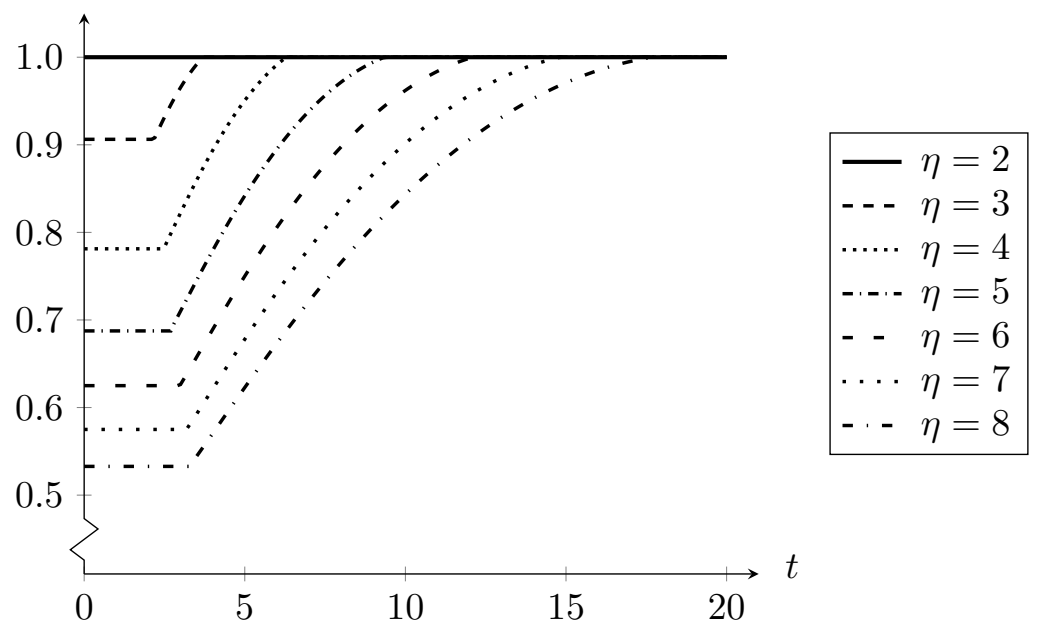

Figure 6: Numerically computed equilibrium strategies: Examples of equilibrium strategies for CobbDouglas utilities $U(t, d)=-d^{0.5}(d-t)^{0.8}$ and $\mu=1, \Delta=0.1, \epsilon=0.001$.

\section{Concluding Remarks}

We have addressed the strategic arrival times to queueing games with general classes of customer preferences and service times. We have done so by introducing a procedure that suffices as a constructive proof for the existence of an equilibrium but also serves as a general method to numerically compute such equilibrium. For concreteness and brevity, we restricted focus to games where customers with preferences composed of waiting and lateness penalties seek service at a single-server facility that opens at a given point in time, has no closing time, does not allow arrivals prior opening, and serves the customers on a FCFS basis. For such queueing games, we showed that there always exist a uniquely determined symmetric equilibrium where each customer arrives according to a continuous probability distribution that extends over a bounded interval of time, admits a positive

\footnotetext{
${ }^{5}$ The Python script used to compute the illustrated examples is available upon request.
} 
probability of arrival at the opening time 0 , and has an open time interval immediately after time 0 where there are no arrivals. We accompanied the equilibrium characterization with numerically computed examples of symmetric equilibria induced by a non-multilinear utility function.

The equilibrium properties characterized in this paper are qualitatively similar to those shown by existing literature that considered queueing games without closing times and arrivals prior to opening (Glazer and Hassin, 1983, Haviv, 2013, and Platz and Østerdal, 2012). While we did not specify a closing time for the server, and does not allow arrivals prior to opening, the basic approach of the constructive procedure easily carries over to variants of such model assumptions. This is also the case if we were to consider other compositional variants of customer preferences. Specifically, customers may not always be worried about the lateness, but rather about the number of customers who arrived ahead of them. This is the case for concerts or flights with unmarked seats, where there is no actual penalty for tardiness unless other customers have arrived and taken hold of the better seats. This disutility is modelled as an order penalty and was recently introduced by Ravner (2014).

There are several important directions for further research on queueing games. One is to analyze the existence of asymmetric equilibria. For queueing games with no closing time and multilinear waiting and lateness penalties, Juneja and Shimkin (2013) established the existence and uniqueness of the equilibrium without assuming a-priori that the equilibrium is symmetric. While such uniqueness does not necessarily hold for games with closing times, it may extend to games with more general classes of preferences. Another direction is the consideration of heterogeneous (multiclass) customers. This has been considered lately in a queueing context by Guo and Hassin (2012), and in the closely related queueing game in Jain et al. (2011). A third direction is to consider target time preferences similar to Vickrey (1969)'s transportation bottleneck model. As previously mentioned, his models assume that customers have a target time at which they wish to get to their destination, and are accordingly penalized for being too early or too late. The lateness penalty considered in the present paper, is a special case of such preferences where the target time equals the opening time of the facility. A fourth direction is the consideration of queueing games with multiple servers. This has recently been considered by Haviv and Ravner (2015) who examine a multi-server system with no queue buffer, where customers are interested in maximizing the probability of obtaining service. A fifth direction is to extend the equilibrium analysis to queueing games with other queue disciplines. This direction is currently being studied by Breinbjerg and Østerdal (2016) who derive equilibrium arrival times under the last-come, first-serve preemptive (LCFS-PR) discipline. The equilibrium analysis in all the aforementioned extended queueing games presents a considerable challenge for future work. 


\section{A Appendix}

\section{A.1 Proof of Proposition 1}

We use standard notation for random variables and event probabilities. That is, for any random variable $\mathbf{Z}$, we refer to $[\mathbf{Z} \leq z]$ as the event that contains all outcomes for which the random variable $\mathbf{Z}$ takes on values lower than $z$. We refer to $\operatorname{Pr}\{\mathbf{Z} \leq z\}$ as the probability of realizing the event $[\mathbf{Z} \leq z]$.

We start the proof by defining some relevant queueing relations for our system. Fix a strategy $F$. Let $\mathbf{A}_{F}(t)$ denote the cumulative arrival process that represents the (random) number of customers who have arrived at the facility in the time interval $[0, t]$ when $\eta-1$ customers arrive independently according to $F$. The process can be expressed as $\mathbf{A}_{F}(t)=\sum_{i=1}^{\eta-1} \mathbb{1}_{\left\{\mathbf{T}_{i} \leq t\right\}}$ where the $\mathbf{T}_{i}$ 's are an independent random variables distributed as $F$ and $\mathbb{1}_{\{\cdot\}}$ denotes the indicator function of any set $\{\cdot\}$. The process is defined on the state space $\{0,1, \ldots, \eta-1\}$ and is right-continuous with left hand limits at all $t$. The process has the initial condition $\mathbf{A}_{F}(0)$ which is a binomial distributed random variable wrt. each customer's probability $F(0)$ of arriving at time 0 . In what follows, we simply denote $\mathbf{A}_{F}(t)$ by $\mathbf{A}(t)$ although the process is always determined by strategy $F$.

We next elaborate on the relation between the arrival process and the customers' departure times. Let $\left\{\mathbf{S}_{j}\right\}_{j \geq 1}$ denote the i.i.d. service time distribution, where $\mathbf{S}_{j}$ is the service time of the customer who is the $j$ th to be served. Let $\mathbf{C}(t)$ denote the number of service completions if the server is busy for $t \geq 0$ time units, so

$$
\mathbf{C}(t)=\max _{m \geq 0}\left\{\sum_{j=1}^{m} \mathbf{S}_{j} \leq t\right\}
$$

assuming that there are infinitely many customers (we are not restricting $\mathbf{C}(t)$ to $\eta-1$ for notational convenience). Let $\mathbf{I}(t)$ denote the idle process at time $t$ which measures the time that the service facility has been idle - or equivalently, the time that the queue has been empty since FCFS is work-conserving - in the time interval $[0, t]$. For each $t$, the value of $t-\mathbf{I}(t)$ is thus the time for which the queue was busy. Let $\mathbf{Q}(t)$ denote the queue length process where $\mathbf{Q}(t)$ is the number of customers in the queue at time $t$ (including the one in service). Formally, the queue length and idle process satisfies the following sample path relation:

$$
\begin{aligned}
\mathbf{Q}(t) & =\mathbf{A}(t)-\mathbf{C}(t-\mathbf{I}(t)) \\
\mathbf{I}(t) & =\int_{0}^{t} \mathbb{1}_{\{\mathbf{Q}(s)=0\}} \mathrm{d} s
\end{aligned}
$$

It follows immediately that $\mathbf{Q}(0)=\mathbf{A}(0)$ once we note that $[\mathbf{I}(0)=0]$ with probability 1. Note also that for any point $t$ where $F(t)$ has an upwards jump, then $\mathbf{A}(t)$ may have a jump discontinuity greater than size 1 and $\mathbf{Q}(t)$ thus has a jump discontinuity of equal 
magnitude to $\mathbf{A}(t)$. Let $\left\{\mathbf{J}_{j}\right\}_{j \geq 1}$ denote the arrival epoch sequence where $\mathbf{J}_{j}$ is the arrival time of the $j$ th customer who arrived at the facility, i.e. $\mathbf{A}(t)=\sup \left\{j: \mathbf{J}_{j} \leq t\right\}$. Let $\mathbf{R}(t)$ denote the residual service time process where $\mathbf{R}(t)$ is the remaining amount of time needed to complete the service of the customer that is in the process of being served at time $t$. Formally,

$$
\mathbf{R}(t)=\mathbf{J}_{\mathbf{A}(t)-\mathbf{Q}(t)+1}+\mathbf{S}_{\mathbf{A}(t)-\mathbf{Q}(t)+1}-t
$$

where the event $[\mathbf{R}(t)=0]$ occurs with probability 1 if $[\mathbf{Q}(t)=0]$ or $[\mathbf{A}(t)=0]$ occurs with probability 1 . For any customer $i \in \mathcal{N}$, let $\mathbf{D}_{i}(t)$ denote the departure time of customer $i$, i.e. the (random) point in time that customer $i$ departs the system if she arrives at time $t$ with certainty. The departure time thus satisfies the following sample path relation:

$$
\mathbf{D}_{i}(t)= \begin{cases}\sum_{j=1}^{\mathbf{X}+1} \mathbf{S}_{j} & , t=0 \\ t+\mathbf{R}(t)+\sum_{j=\mathbf{A}(t)-\mathbf{Q}(t)+2}^{\mathbf{A}(t)-\mathbf{Y}} \mathbf{S}_{j}+\mathbf{S}_{\mathbf{A}(t)+1} & , t>0 \text { where } F_{-i}(t) \text { is discontinuous } \\ t+\mathbf{R}(t)+\sum_{j=\mathbf{A}(t)-\mathbf{Q}(t)+2}^{\mathbf{A}(t)} \mathbf{S}_{j}+\mathbf{S}_{\mathbf{A}(t)+1} & , t>0 \text { where } F_{-i}(t) \text { is continuous }\end{cases}
$$

where $\mathbf{X} \sim \operatorname{unif}\{0,1, \ldots, \mathbf{Q}(0)\}$ and $\mathbf{Y} \sim \operatorname{unif}\left\{0,1, \ldots, \mathbf{Q}(t)-\lim _{s \uparrow t} \mathbf{Q}(s)\right\}$ for which unif $\{\cdot\}$ denotes a uniform distribution of any discrete set $\{\cdot\}$. Note that the empty sum applies in cases where the lower bound of summation exceeds the upper bound. Intuitively, $\mathbf{D}_{i}(t)$ depends on points of discontinuity in $F$ due to the possibility of simultaneous arrivals at such points in time. Recall that customers who arrive simultaneously is admitted for service in a uniformly randomized order. The random variables $\mathbf{X}$ and $\mathbf{Y}$ thus represents the resulting service order of customer $i$ under such uniform lotteries. Conversely, the probability of simultaneous arrivals equals zero at all points in time where $F$ is continuous, hence the service order of customer $i$ is strict. According to these definitions, all processes are right-continuous with left limits. Note that the cumulative departure time distribution $D_{i}(d \mid t, F)$ is defined by $\operatorname{Pr}\left\{\mathbf{D}_{i}(t) \leq d\right\}$.

We are now ready to prove the claims of Proposition 1 . We prove each claim seperately:

Claim $(i)$ in Proposition 1 follows immediately from the sample path relation of $\mathbf{D}_{i}(t)$ since the independent and identical distributed service time $\mathbf{S}_{j}$ for all $j \geq 1$ are continuous by definition.

Claim (ii) follows by the sample path relation (12). To see this, note that $\mathbf{A}(\cdot)$ is a everywhere right-continuous and non-decreasing counting process. For any point in time $t$ that $\mathbf{A}(t)$ has an upward jump, then $\mathbf{Q}(t)$ has an upwards jump of equal magnitude since the busy time $t-\mathbf{I}(t)$ is a continuous process. Clearly, by relation (12), $\mathbf{D}_{i}(t)$ also has an upwards jump at $t$ since $\mathbf{R}(t)$ is continuous at $t$, hence the claim follows immediately as any customer could arrive immediately before time $t$ and almost surely depart the system earlier than if she arrive at time $t$. 
Claim (iii) follows immediately from the sample path relation of $\mathbf{D}_{i}(t)$ once we note that customers who arrive simultaneously at time 0 are admitted into service in a uniformly randomized order, while a customer arriving immediately after zero will have to wait for the service completion of the customers arriving at zero.

Claim (iv) follows by a stochastic coupling argument. Let two arrival processes $\mathbf{A}$ and $\tilde{\mathbf{A}}$ be implemented on common probability space, and let all customers have identical services times. With the assumed dominance relation stated in claim (iv), it follows that $\mathbf{A}(s)=\tilde{\mathbf{A}}(s)$ for all $0 \leq s<t$ and $\mathbf{A}(t) \geq \tilde{\mathbf{A}}(t)$. This implies that $\mathbf{Q}(t) \geq \tilde{\mathbf{Q}}(t)$ with probability 1 . From sample path relation (12), it follows that $\mathbf{D}_{i}(t) \geq \tilde{\mathbf{D}}_{i}(t)$ with probability 1 for any $i$.

\section{A.2 Proof of Lemma 6}

Applying the same notation of the queueing relations as defined in Appendix A.1, we prove each part of Lemma 6 separately. In both cases, we use stochastic coupling with two arrival processes implemented on common probability space, and with identical service times for all customers (see Juneja and Shimkin, 2013).

We first consider part $(i)$. For any customer $i \in \mathcal{N}$, note that $\mathbf{A}\left(s_{2}\right)-\mathbf{A}\left(s_{1}\right)=$ $\sum_{j \in \mathcal{N} \backslash\{i\}} \mathbb{1}_{\left\{s_{1}<\mathbf{T}_{j} \leq s_{2}\right\}}$ for $s_{1}<s_{2}$ where $\mathbf{T}_{j} \sim F$. It follows from the assumed dominance relation in part $(i)$ that $\mathbf{A}\left(s_{2}\right)-\mathbf{A}\left(s_{1}\right) \leq \tilde{\mathbf{A}}\left(s_{2}\right)-\tilde{\mathbf{A}}\left(s_{1}\right)$ for all $s_{1}<s_{2} \leq t$. This implies that $\mathbf{Q}(t) \leq \tilde{\mathbf{Q}}(t)$ with probability 1. From sample path relation (12), it follows that $\mathbf{D}_{i}(t) \leq \tilde{\mathbf{D}}_{i}(t)$ with probability 1 , hence proving part $(i)$ of Lemma 6 .

As for the claim of part (ii), with the same coupling, it follows from the assumed dominance relation in (ii) that $\mathbf{Q}(t) \geq \tilde{\mathbf{Q}}(t)$ with probability 1 which implies that $\mathbf{D}_{i}(t) \geq$ $\tilde{\mathbf{D}}_{i}(t)$ with probability 1 . To see this, let $t^{\prime}=\max \{s \leq t \mid F(s)=\tilde{F}(s)\}$ and note that for any interval $s_{1}<s_{2} \leq t$ where $\mathbf{A}\left(s_{2}\right)-\mathbf{A}\left(s_{1}\right) \geq \tilde{\mathbf{A}}\left(s_{2}\right)-\tilde{\mathbf{A}}\left(s_{1}\right)$ it implies that $\mathbf{Q}\left(s_{2}\right)-\mathbf{Q}\left(s_{1}\right) \geq \tilde{\mathbf{Q}}\left(s_{2}\right)-\tilde{\mathbf{Q}}\left(s_{1}\right)$ with probability 1. Since $\mathbf{A}\left(t^{\prime}\right)=\tilde{\mathbf{A}}\left(t^{\prime}\right)$ and $\mathbf{A}(s) \leq \tilde{\mathbf{A}}(s)$ for all $0 \leq s<t^{\prime}$, by assumption, there exists a time interval $s<t^{\prime} \leq t$ such that $\mathbf{Q}\left(t^{\prime}\right)-\mathbf{Q}(s) \geq \tilde{\mathbf{Q}}\left(t^{\prime}\right)-\tilde{\mathbf{Q}}(s)$ where $\mathbf{Q}\left(t^{\prime}\right) \geq \tilde{\mathbf{Q}}\left(t^{\prime}\right)$ with probability 1 , hence proving part (ii) of Lemma 6.

\section{A.3 Procedure for computing $\mathcal{X}_{l}$}

In this section, we first formulate the transient dynamics of the stochastic queueing system under a given strategy $F$. We next present a numerical procedure that applies the transient dynamics in order to compute the function $X_{l}$ for a given value of $0 \leq l \leq 1$. Lastly, we derive the computational complexity of the procedure.

Fix a strategy $F$. Assume also that customer service times are independently, identically and exponentially distributed with rate $\mu$, and that $F$ does not have any points of upward discontinuity. For any $i \in \mathcal{N}$, we start the transient analysis by establishing the 
probability of how many customers arrive at the opening time 0 when $\eta-1$ customers arrive according to $F$. Let $\mathbf{A}(t)$ be the cumulative arrival process determined by $F$ and note that $\operatorname{Pr}\{\mathbf{A}(0)=a\}$ is a binomial distribution with $\eta-1$ independent customers, each of which has the probability $F(0)$ of arriving at time 0. Formally,

$$
\operatorname{Pr}\{\mathbf{A}(0)=a\}= \begin{cases}\left(\begin{array}{c}
\eta-1 \\
a
\end{array}\right) F(0)^{a}(1-F(0))^{\eta-1-a} & \text { for } F(0)>0 \\
1 & \text { for } F(0)=0 \text { and } a=0 . \\
0 & \text { for } F(0)=0 \text { and } a \neq 0\end{cases}
$$

We next address the dynamics of $\operatorname{Pr}\left\{\mathbf{D}_{i}(t) \leq d\right\}$ immediately after the opening time 0 where the service facility commences operation. Some care is required in formulating the evolution of $\operatorname{Pr}\left\{\mathbf{D}_{i}(t) \leq d\right\}$ over time $t>0$ since the arrival process is not memoryless, and the arrival intensity depends on the customers that already arrived. The system can be presented as a time-inhomogeneous, two dimensional Markov chain $\{(\mathbf{A}(t), \mathbf{Q}(t))\}_{t \in \mathbb{R}_{0}^{+}}$ with transient states $(a, q)$ where $a=0,1, \ldots, \eta-1$ is the number of customers that have arrived and $q=0,1, \ldots, \eta-1$ is the number of customers in the queue. Let $p_{a, q}(t)=$ $\operatorname{Pr}\{\mathbf{A}(t)=a, \mathbf{Q}(t)=q\}$ denote the joint probability of state $(a, q)$ at time $t$. Suppose that $F$ admits a density $F^{\prime}$ and recall that $H(t)=F^{\prime}(t) /(1-F(t))$ is the hazard rate function associated with $F$. Using the Kolmogorov forward equations, the dynamics of the Markov formulation are summarized in the following lemma.

Lemma 8 Let $F$ be a strategy for any queueing game $\mathcal{G}$. For any $i \in \mathcal{N}$ and some point in time $t_{a} \geq 0$, then

$$
\begin{aligned}
\frac{\mathrm{d}}{\mathrm{d} t} p_{a, 0}(t)= & \mu p_{a, 1}(t)-(\eta-1-a) H(t) p_{a, 0}(t), \quad 0 \leq a \leq \eta-1 \\
\frac{\mathrm{d}}{\mathrm{d} t} p_{a, q}(t)= & \mu p_{a, q+1}(t)+(\eta-a) H(t) p_{a-1, q-1}(t) \\
& -(\mu+(\eta-1-a) H(t)) p_{a, q}(t), \quad 1 \leq q \leq a \leq \eta-1
\end{aligned}
$$

for all $t \geq t_{a}$ and the initial condition is $p_{a, q}\left(t_{a}\right)$ where $t_{a}=\inf \left\{t \mid t>0: U_{i}(0, F) \leq\right.$ $\left.U_{i}(t, F)\right\}$.

Remark 1. The equations above can be easily modified when the density of $F^{\prime}(t)$ is not well defined everywhere, by writing $\mathrm{d} F(t)$ in place of $F^{\prime}(t) \mathrm{d} t$ within $H(t)$.

Suppose for now that there exists an interior hole $\left(0, t_{a}\right)$ within the support $\mathcal{S}(F)$. Then the initial condition $p_{a, q}\left(t_{a}\right)$ depends on how many customers have been served during the time interval $\left(0, t_{a}\right)$. In particular, the number of customers possibly served by the service facility within $\left(0, t_{a}\right)$ follows a Poisson distribution with parameter $\mu t$. We use the term "possibly" since the system might be empty during $\left(0, t_{a}\right)$, or part of it, and hence, although service is ready to be provided, there is no one there to enjoy it. One 
more thing to observe here is that if one stops the clock when the server is idle, then the departure process under the new clock is a pure Poisson death process. Let $C_{a, q}(t)$ denote the probability that exactly $a-q$ customers have been served at time $t$ during time interval $(0, t]$ assuming that no customers arrive in this interval. Formally,

$$
C_{a, q}(t)= \begin{cases}1 & \text { for } a=q=0 \\ 1-\sum_{k=1}^{a} \frac{e^{-\mu t}(\mu t)^{a-k}}{(a-k) !} & \text { for } a>q=0 \\ \frac{e^{-\mu t}(\mu t)^{a-q}}{(a-q) !} & \text { for } a \geq q>0 \\ 0 & \text { for } q>a \geq 0\end{cases}
$$

where for $a>q=0$, the probability $C_{a, 0}(t)$ is the complementary event. The joint probability $p_{a, q}(t)$ is thus defined as

$$
p_{a, q}(t)=C_{a, q}(t) \operatorname{Pr}\{\mathbf{A}(0)=a\} \quad \text { for all } t \leq t_{a}
$$

where $t_{a}=\inf \left\{t \mid t>0: U_{i}(0, F) \leq U_{i}(t, F)\right\}$ for any $i$. The initial condition of the difference-differential equation in Lemma 8 is thus $p_{a, q}\left(t_{a}\right)$ as defined in (14).

With the Markov formulation above, we then need to characterize the cumulative departure times distribution $D_{i}(d \mid t, F)$ for all $t \in \mathbb{R}_{0}^{+}$in order to evaluate $U_{i}(t, F)$. The following lemma establishes such characterization of $D_{i}$ :

Lemma 9 Let $F$ be an equilibrium strategy for any queueing game $\mathcal{G}$ such that $F$ has no points of upward discontinuity. Then the cumulative departure time distribution $D_{i}$ for any $i \in \mathcal{N}$ is defined as,

$$
D_{i}(d \mid t, F)= \begin{cases}\sum_{a=0}^{\eta-1} \sum_{x=0}^{a} \frac{E(d, x+1, \mu)}{a+1} \operatorname{Pr}\{\mathbf{A}(0)=a\} & \text { for } t=0 \\ \sum_{a=0}^{\eta-1} \sum_{q=0}^{a} E(d-t, q+1, \mu) p_{a, q}(t) & \text { for } t>0\end{cases}
$$

where $\operatorname{Pr}\{\mathbf{A}(0)=a\}$ is defined by eq. (13), $p_{a, q}(t)$ is defined by the Markov formulation in Lemma 8, and $E$ is the cumulative Erlang distribution defined by

$$
E(d, q, \mu)= \begin{cases}1-\sum_{k=0}^{q-1} \frac{e^{-\mu d}(\mu d)^{k}}{k !} & \text { for } d \geq t \\ 0 & \text { for } d<t\end{cases}
$$

Proof. We start by establishing the probability of departure times when some customer $i$ arrives at time 0 . Recall that the departure time $\mathbf{D}_{i}(0)$ is determined by the number of customers $\mathbf{A}(0)$ (customer $i$ excluded) who also arrive at $t=0$, and $i$ 's service order $\mathbf{X}$ which is uniformly distributed over the number of customers who arrived simultaneously at 0 . Then $\operatorname{Pr}\left\{\mathbf{D}_{i}(0) \leq d\right\}$ is marginal probability when summing all possible configurations of the other events $[\mathbf{A}(0)=a, \mathbf{X}=x]$ such that 


$$
\begin{aligned}
\operatorname{Pr}\left\{\mathbf{D}_{i}(0) \leq d\right\} & =\sum_{a=0}^{\eta-1} \sum_{x=0}^{\eta-1} \operatorname{Pr}\left\{\mathbf{A}(0)=a, \mathbf{X}=x, \mathbf{D}_{i}(0) \leq d\right\} \\
& =\sum_{a=0}^{\eta-1} \sum_{x=0}^{a} \operatorname{Pr}\left\{\mathbf{D}_{i}(0) \leq d \mid \mathbf{A}(0)=a, \mathbf{X}=x\right\} \operatorname{Pr}\{\mathbf{X}=x \mid \mathbf{A}(0)=a\} \operatorname{Pr}\{\mathbf{A}(0)=a\} \\
& =\sum_{a=0}^{\eta-1} \sum_{x=0}^{a} \operatorname{Pr}\left\{\sum_{j=1}^{x+1} \mathbf{S}_{j} \leq d \mid \mathbf{A}(0)=a, \mathbf{X}=x\right\} \frac{1}{a+1} \operatorname{Pr}\{\mathbf{A}(0)=a\} \\
& =\sum_{a=0}^{\eta-1} \sum_{x=0}^{a} E(d, x+1, \mu) \frac{1}{a+1} \operatorname{Pr}\{\mathbf{A}(0)=a\} .
\end{aligned}
$$

The second equality follows by the theorem of iterated conditional probability when we exclude event configurations of zero probability, i.e. every event $[\mathbf{A}(0)=a, \mathbf{X}=x]$ where $x>a$. The third equality follows immediately by the definition of $\mathbf{D}_{i}(0)$ and the probability mass function of $\mathbf{X}$ which is uniformly distributed over $\{0, \ldots, a\}$ conditional on the event $[\mathbf{A}(0)=a]$. The last equality follows immediately, once we note that the sum of independent, exponentially distributed random variables with identical rate parameter $\mu$ follows an Erlang distribution.

With the Markov formulation from Lemma 8, we characterize the marginal probability of departure $\operatorname{Pr}\left\{\mathbf{D}_{i}(t) \leq d\right\}$ when summing all possible configurations of the events $[\mathbf{A}(t)=a, \mathbf{Q}(t)=q]$ such that for $t>0$,

$$
\begin{aligned}
\operatorname{Pr} & \left\{\mathbf{D}_{i}(t) \leq d\right\}=\sum_{a=0}^{\eta-1} \sum_{q=0}^{\eta-1} \operatorname{Pr}\left\{\mathbf{A}(t)=a, \mathbf{Q}(t)=q, \mathbf{D}_{i}(t) \leq d\right\} \\
& =\sum_{a=0}^{\eta-1} \sum_{q=0}^{a} \operatorname{Pr}\left\{\mathbf{D}_{i}(t) \leq d \mid \mathbf{A}(t)=a, \mathbf{Q}(t)=q\right\} \operatorname{Pr}\{\mathbf{Q}(t)=q \mid \mathbf{A}(t)=a\} \operatorname{Pr}\{\mathbf{A}(t)=a\} \\
& =\sum_{a=0}^{\eta-1} \sum_{q=0}^{a} \operatorname{Pr}\left\{t+\mathbf{R}(t)+\sum_{j=a-q+2}^{a} \mathbf{S}_{j}+\mathbf{S}_{a+1} \leq d \mid \mathbf{A}(t)=a, \mathbf{Q}(t)=q\right\} \operatorname{Pr}\{\mathbf{A}(t)=a, \mathbf{Q}(t)=q\} \\
& =\sum_{a=0}^{\eta-1} \sum_{q=0}^{a} \operatorname{Pr}\left\{t+\sum_{j=1}^{q+1} \mathbf{S}_{j} \leq d \mid \mathbf{A}(t)=a, \mathbf{Q}(t)=q\right\} p_{a, q}(t) \\
& =\sum_{a=0}^{\eta-1} \sum_{q=0}^{a} E(d-t, q+1, \mu) p_{a, q}(t) .
\end{aligned}
$$

The second equality follows by the theorem of iterated conditional probability when we exclude event configurations of zero probability, i.e. every event $[\mathbf{A}(t)=a, \mathbf{Q}(t)=q]$ where $q>a$. The third equality follows immediately by Bayes' theorem and the definition of $\mathbf{D}_{i}(0)$. The fourth equality follows from the memoryless property of the independently and exponentially distributed service times, which implies that $\mathbf{R}(t)$ is exponentially distributed. The last equality follows immediately, once we note that $t+\sum_{j} \mathbf{S}_{j}$ follows an Erlang distribution. Combining the separate expressions for $\operatorname{Pr}\left\{\mathbf{D}_{i}(0) \leq d\right\}$ and $\operatorname{Pr}\left\{\mathbf{D}_{i}(t) \leq d\right\}$, Lemma 9 follows immediately. 


\section{Numerical Procedure}

We now present a numerical method to compute $X_{l}$ for a given $l$. Note that $U, \eta, \mu, \epsilon, \Delta$ and $l$ are exogenous inputs to the procedure:

(i) Let $\mathcal{T}_{\Delta}=\{t \in \mathbb{N}: t \Delta\}$ be a discretization of $\mathbb{R}_{0}^{+}$wrt. $\Delta$.

(ii) Fix $X_{l, h}(0)=l$ for all $h=0,1, \ldots$

(iii) Compute $U_{i}\left(0, X_{l, h}\right)=\int_{0}^{\infty} U(0, d) \mathrm{d} D_{i}\left(d \mid 0, X_{l, h}\right)$ according to Lemma 9 (note that $U_{i}\left(0, X_{l, h}\right)$ is the same for all $\left.h\right)$.

(iv) Compute $t_{a}=\inf \left\{t \in \mathcal{T} \mid t>0: U_{i}\left(0, X_{l, h}\right) \leq U_{i}\left(t, X_{l, h}\right)\right\}$ according to Lemma 9 with $p_{a, q}(t)$ defined by (14).

(v) If $l=1$ or $t_{a}=\infty$, let $X_{l}(t)=l$ for all $t \in \mathcal{T}_{\Delta}$ and stop procedure.

(vi) Let $X_{l, h}(t)=l$ for all $t \in \mathcal{T}_{\Delta}$ where $0 \leq t \leq t_{a}$.

(vii) Let $h=0$ and sequentially compute $X_{l, 0}(t)$ for $t \in \mathcal{T}_{\Delta}$ and $t \geq t_{a}$ according to equation (2).

The approximation of $p_{a, q}$ from one time step to another is given by the Kolmogorov equations in Lemma 8 with the initial condition $p_{a, q}\left(t_{a}\right)$. We consider two distinct cases of approximation steps:

First, let $t_{a}^{\Delta}=\left\lceil\frac{t_{a}}{\Delta}\right\rceil \Delta$ denote the smallest discretised time step in $\mathcal{T}_{\Delta}$ subsequent to $t_{a}$ where $\lceil\cdot\rceil$ is the ceiling function that maps a real number to the smallest following integer. Then the approximation step of $p_{a, q}\left(t_{a}\right)$ from $t_{a}$ to $t_{a}^{\Delta}$ is given by

$$
\begin{aligned}
p_{a, 0}\left(t_{a}^{\Delta}\right)= & p_{a, 0}\left(t_{a}\right)+\left(t_{a}^{\Delta}-t_{a}\right) \mu p_{a, 1}\left(t_{a}\right)-\left(t_{a}^{\Delta}-t_{a}\right)(\eta-1-a) H\left(t_{a}\right) p_{a, 0}\left(t_{a}\right), 0 \leq a \leq \eta-1 \\
p_{a, q}\left(t_{a}^{\Delta}\right)= & p_{a, q}\left(t_{a}\right)+\left(t_{a}^{\Delta}-t_{a}\right) \mu p_{a, q+1}\left(t_{a}\right)+\left(t_{a}^{\Delta}-t_{a}\right)(\eta-a) H\left(t_{a}\right) p_{a-1, q-1}\left(t_{a}\right) \\
& -\left(t_{a}^{\Delta}-t_{a}\right)\left(\mu+(\eta-1-a) H\left(t_{a}\right)\right) p_{a, q}\left(t_{a}\right), \quad 1 \leq q \leq a \leq \eta-1
\end{aligned}
$$

where $H\left(t_{a}\right)=\frac{X_{l, 0}\left(t_{a}^{\Delta}\right)-l}{t_{a}^{\Delta}-t_{a}} /(1-l)$ according to the condition in equation (3).

Second, the approximation step of $p_{a, q}(t)$ from $t$ to $t+\Delta$ where $t \geq t_{a}^{\Delta}$ is given by

$$
\begin{aligned}
p_{a, 0}(t+\Delta)= & p_{a, 0}^{0}(t)+\Delta \mu p_{a, 1}^{0}(t)-\Delta(\eta-1-a) H(t) p_{a, 0}^{0}(t), \quad 0 \leq a \leq \eta-1 \\
p_{a, q}(t+\Delta)= & p_{a, q}^{0}(t)+\Delta \mu p_{a, q+1}^{0}(t)+\Delta(\eta-a) H(t) p_{a-1, q-1}^{0}(t) \\
& -\Delta(\mu+(\eta-1-a) H(t)) p_{a, q}^{0}(t), \quad 1 \leq q \leq a \leq \eta-1
\end{aligned}
$$

where $H(t)=\frac{X_{l, 0}(t+\Delta)-l}{\Delta} /(1-l)$ and $p_{a, q}^{0}(t)=C_{a, q}(t) \operatorname{Pr}\{\mathbf{A}(0)=a\}$ according to the condition in equation (3).

Stop at the first time step $t^{\prime} \in \mathcal{T}_{\Delta}$ such that either of the following two conditions are met:

(a) $\left|1-X_{l, 0}\left(t^{\prime}\right)\right| \leq \epsilon$ 
(b) $\sum_{a=0}^{\eta-1} p_{a, 0}^{0}\left(t^{\prime}\right) \geq 1-\epsilon$

Compute $\bar{X}_{l, 0}(t)$ according to equation (4) for all $t \in \mathcal{T}_{\Delta}$.

(viii) Assign $h:=h+1$ and sequentially compute $X_{l, h}(t)$ for $t \in \mathcal{T}_{\Delta}$ and $t \geq t_{a}$ according to equation (5).

Similarly to step (vii), the approximation step of $p_{a, q}$ is given by the Kolmogorov equations. That is, the approximation step of $p_{a, q}\left(t_{a}\right)$ from $t_{a}$ to $t_{a}^{\Delta}$ is given as in step (vii) with $X_{l, h}$ in place of $X_{l, 0}$ in the hazard rate function $H\left(t_{a}\right)$. Moreover, the approximation step of $p_{a, q}(t)$ from $t$ to $t+\Delta$ where $t \geq t_{a}^{\Delta}$ is given by

$$
\begin{aligned}
p_{a, 0}(t+\Delta)= & p_{a, 0}^{h}(t)+\Delta \mu p_{a, 1}^{h}(t)-\Delta(\eta-1-a) H(t) p_{a, 0}^{h}(t), \quad 0 \leq a \leq \eta-1 \\
p_{a, q}(t+\Delta)= & p_{a, q}^{h}(t)+\Delta \mu p_{a, q+1}^{h}(t)+\Delta(\eta-a) H(t) p_{a-1, q-1}^{h}(t) \\
& -\Delta(\mu+(\eta-1-a) H(t)) p_{a, q}^{h}(t), \quad 1 \leq q \leq a \leq \eta-1
\end{aligned}
$$

where $H(t)=\frac{X_{l, h}(t+\Delta)-\bar{X}_{l, h-1}(t)}{\Delta} /\left(1-\bar{X}_{l, h-1}(t)\right)$ and the approximation step of $p_{a, q}^{h}(t)$ from $t$ to $t+\Delta$ is given by

$$
\begin{aligned}
p_{a, 0}^{h}(t+\Delta)= & p_{a, 0}^{h}(t)+\Delta \mu p_{a, 1}^{h}(t)-\Delta(\eta-1-a) H_{h}(t) p_{a, 0}^{h}(t), 0 \leq a \leq \eta-1 \\
p_{a, q}^{h}(t+\Delta)= & p_{a, q}^{h}(t)+\Delta \mu p_{a, q+1}^{h}(t)+\Delta(\eta-a) H_{h}(t) p_{a-1, q-1}^{h}(t) \\
& -\Delta\left(\mu+(\eta-1-a) H_{h}(t)\right) p_{a, q}^{h}(t), \quad 1 \leq q \leq a \leq \eta-1
\end{aligned}
$$

with the initial condition $p_{a, q}^{h}\left(t_{a}^{\Delta}\right)=p_{a, q}\left(t_{a}^{\Delta}\right)$ and $H_{h}(t)=\frac{\bar{X}_{l, h-1}(t+\Delta)-\bar{X}_{l, h-1}(t)}{\Delta} /(1-$ $\left.\bar{X}_{l, h-1}(t)\right)$ according to the condition in equation (6).

Stop at the first time step $t^{\prime} \in \mathcal{T}_{\Delta}$ such that either of the following two conditions are met:

(a) $\left|1-X_{l, h}\left(t^{\prime}\right)\right| \leq \epsilon$

(b) $\sum_{a=0}^{\eta-1} p_{a, 0}^{h}\left(t^{\prime}\right) \geq 1-\epsilon$

Compute $\bar{X}_{l, h}(t)$ according to equation (7) for all $t \in \mathcal{T}_{\Delta}$.

(ix) If $\bar{X}_{l, h}(t)-\bar{X}_{l, h-1}(t) \leq \epsilon$ for all $t \in \mathcal{T}_{\Delta}$, let $X_{l}=\bar{X}_{l, h}$ and stop procedure.

(x) Else, go back to (viii) and begin the next iteration of $h$

Remark 2. The stopping conditions (a) and (b) approximates (wrt. the tolerance parameter $\epsilon$ ) the time step at which $X_{l, h}$ has reach its global maximum for each $h$ with certainty. That is, if either (a) $\left|1-X_{l, h}\left(t^{\prime}\right)\right| \leq \epsilon$ or (b) $\sum_{a=0}^{\eta-1} p_{a, 0}^{h}\left(t^{\prime}\right) \geq 1-\epsilon$ holds for some time step $t^{\prime} \in \mathcal{T}_{\Delta}$, then $\left|X_{l, h}(t)-X_{l, h}\left(t^{\prime}\right)\right| \leq \epsilon$ for all $t \geq t^{\prime}$ by construction. This follows immediately, once we note that $X_{l, h}$ is bounded from above at 1 , and moreover, that $U$ is strictly monotonically decreasing in the departure time. 


\section{Computational Complexity}

This section addresses the computational complexity of the numerical procedure presented above. For a given value of $l$, recall that the procedure computes $X_{l}$ by recursively computing $X_{l, h}$ through (possibly multiple) iterations of $h$ until $X_{l, h}$ converges to $X_{l}$. In particular, any $X_{l}$ for which $l<1$ or $t_{a}<\infty$ requires multiple recursive iterations of $h$ for $X_{l, h}$ to converge. The following lemma establishes the computational complexity of the numerical procedure for each recursive iteration of $h$ and a given value of $l$.

Lemma 10 For any queueing game $\mathcal{G}$ with i.i.d. exponential customer service times, the computational complexity of the numerical procedure for each iteration of $l$ and $h$ with $\eta$ customers and parameters $\Delta$ and $\epsilon$ is

$$
\mathcal{O}\left(\frac{T_{l, h}(\epsilon) \eta^{2}}{2 \Delta}\right)
$$

where $T_{l, h}$ is the stopping rule defined as

$$
T_{l, h}(\epsilon)=\min \left\{\inf \left\{t \mid X_{l, h}(t) \geq 1-\epsilon\right\}, \min \left\{t \mid \sum_{a=0}^{\eta-1} p_{a, q}^{h}(t) \geq 1-\epsilon\right\}\right\}
$$

with $X_{l, h}$ and $p_{a, q}^{h}$ defined in the numerical procedure's step (vii) and (viii).

Proof. For a given value of $l$ and $h$, the number of partitions of the interval $\left[0, T_{l, h}\right]$ in the discretization is

$$
\frac{T_{l, h}+1}{\Delta} .
$$

In each partition, the probability of all states of the Markov process $\{(\mathbf{A}(t), \mathbf{Q}(t))\}_{t \in \mathbb{R}_{0}^{+}}$is approximated. The size of the state space is

$$
|\{(a, q): 0 \leq a \leq \eta-1, a \leq q \leq \eta-1\}|=\frac{\eta(\eta+1)}{2} .
$$

The total number of approximations in the procedure is thus

$$
\frac{\eta(\eta+1)\left(T_{l, h}+1\right)}{2 \Delta}
$$

The claim in Lemma 10 then follows immediately from the limit behavior of (15) as the number of customers $\eta$ becomes extremely large.

\section{References}

Breinbjerg, J. and Østerdal, L. P. (2016). Equilibrium arrival times in queueing games: Welfare comparisons under the FCFS and LCFS-PR discipline. Unpublished manuscript.

Breinbjerg, J., Sebald, A., and Østerdal, L. P. (2016). Strategic behavior and social outcomes in a bottleneck queue: Experimental evidence. Review of Economic Design, DOI: 10.1007/s10058-016-0190-4. 
Glazer, A. and Hassin, R. (1983). ?/M/1: On the equilibrium distribution of customer arrivals. European Journal of Operational Research, 13(2):146-150.

Glazer, A. and Hassin, R. (1987). Equilibrium arrivals in queues with bulk service at scheduled times. Transportation Science, 21(4):273-278.

Guo, P. and Hassin, R. (2012). Strategic behavior and social optimization in markovian vacation queues: The case of heterogeneous customers. European Journal of Operational Research, 222(2):278-286.

Hassin, R. (2016). Rational Queueing. Chapman and Hall/CRC.

Hassin, R. and Haviv, M. (2003). To Queue or not to Queue: Equilibrium Behavior in Queueing Systems. (Vol. 59). Springer.

Hassin, R. and Kleiner, Y. (2011). Equilibrium and optimal arrival patterns to a server with opening and closing times. IIE Transactions, 43(3):820-827.

Haviv, M. (2013). When to arrive at a queue with tardiness costs? Performance Evaluation, 70(6):387-399.

Haviv, M. and Ravner, L. (2015). Strategic timing of arrivals to a finite queue multi-server loss system. Queueing Systems, 81(1):71-96.

Jain, R., Juneja, S., and Shimkin, N. (2011). The concert queueing game: To wait or to be late. Discrete Event Dynamic Systems, 21(1):103-138.

Juneja, S. and Shimkin, N. (2013). The concert queueing game: Strategic arrivals with waiting and tardiness costs. Queueing Systems, 74(4):369-402.

Nash, J. (1951). Non-cooperative games. The Annals of Mathematics, 54(2):286-295.

Platz, T. T. and Østerdal, L. P. (2012). The curse of the first-in-first-out queue discipline. Discussion Papers of Business and Economics, University of Southern Denmark, No. 10/2012.

Rapoport, A., Stein, W. E., Parco, J. E., and Seale, D. A. (2004). Equilibrium play in single-server queues with endogenously determined arrival times. Journal of Economic Behavior and Organization, 55(1):67-91.

Ravner, L. (2014). Equilibrium arrival times to a queue with order penalties. European Journal of Operation Research, 239(2):456-468.

Rothschild, M. and Stiglitz, J. (1970). Increasing risk: I. a definition. Journal of Economic Theory, $2(3): 225-243$.

Seale, D. A., Parco, J. E., Stein, W. E., and Rapoport, A. (2005). Joining a queue or staying out: Effects of information structure and service time on arrival and staying out decisions. Experimental Economics, $8(2): 117-144$.

Stein, W. E., Rapoport, A., Seale, D. A., Zhang, H., and Zwick, R. (2007). Batch queues with choice of arrivals: Equilibrium analysis and experimental study. Games and Economic Behavior, 59(2):345-363.

Vickrey, W. S. (1969). Congestion theory and transportation investment. American Economic Review, $59(2): 251-260$. 